\title{
The role of dendritic cells in the immunopathogenesis of psoriasis
}

\author{
Sunit P. Jariwala
}

Received: 15 March 2007 / Revised: 18 June 2007 / Accepted: 20 July 2007 / Published online: 7 August 2007

(C) Springer-Verlag 2007

\begin{abstract}
Psoriasis vulgaris is a chronic inflammatory skin disease that is marked by a complex interplay of dendritic cells (DCs), T-cells, cytokines, and downstream transcription factors as part of a self-sustaining type 1 cytokine network. As integral players of the immune system, DCs represent antigen-presenting cells that are crucial for efficient activation of T-cells and B-cells. DCs have also been linked to distinct chronic inflammatory conditions, including psoriasis. In the setting of psoriasis therapy, $\mathrm{DC} / \mathrm{T}$ cell interactions serve as a potential target for biologic response modifiers. Here we describe the major DC subsets as well as the immunologic involvement of DCs within the context of psoriatic lesions.
\end{abstract}

\section{Introduction}

The clearance of psoriasis using targeted immunotherapies demonstrates the significant roles of T-cells, dendritic cells (DCs), and cytokines as part of a type 1 inflammatory network [33]. Central to this model are the upstream activation of DCs, DC-induced cytokine production (IL-12, IL-23), and antigen presentation to type $1 \mathrm{~T}$-cells. Subsequently, stimulated T-cells release tumor necrosis factor-alpha (TNF- $\alpha$ ), interferon-gamma (IFN- $\gamma$ ), and other cytokines $[9,19]$. Collectively, TNF- $\alpha$ and IFN- $\gamma$ induce a wide variety of responses including: signal transducer and activator of transcription 1 (STAT1) stimulation followed by expres-

S. P. Jariwala $(\square)$

Department of Internal Medicine, Albert Einstein/Montefiore Medical Center, 33 Teal Court, East Windsor, NJ 08520, USA e-mail: sjariwal@montefiore.org sion of downstream response genes; activation of nuclear factor kappa B (NF- $\kappa \mathrm{B})$ signaling pathways; leukocyte migration to inflammatory regions; and vasodilation that results in the erythema that is characteristic of psoriatic lesions [11, 33, 34, 42]. It has been postulated that TNF- $\alpha$ and IFN- $\gamma$ activate keratinocytes that produce a wide variety of cytokines, which are capable of effecting DC maturation [22]. Chemokines, which are produced through a downstream response to IFN- $\gamma$, TNF- $\alpha$, and possibly other cytokines, also play a role in recruiting leukocytes to areas of inflammation [33, 58]. The noted cytokine, chemokine, and cellular interactions may contribute to the self-perpetuation of the type 1 pathway, which can in turn be correlated with the chronic nature of psoriasis.

DCs are key players in the immune mechanisms surrounding psoriasis, as well as other disease processes. Initially described by Steinman and Cohn in 1973, as identified in the lymphoid organs of mice, DCs have been recognized as antigen presenting cells (APCs) that are crucial for the presentation of molecules to T-cells and B-cells [64]. DCs are bone marrow-derived, circulate in the bloodstream in immature forms, and migrate into peripheral tissues. Immature DCs function in antigen detection, uptake and processing, whereas mature DCs primarily function in antigen presentation as well as cytokine production. DCs have been linked to diseases of chronic inflammation including psoriasis, Sjögren's syndrome, rheumatoid arthritis, systemic lupus erythematosus (SLE), and multiple sclerosis [15]. This paper reviews the role of DCs in the immunopathogenesis of psoriasis, while illustrating: the DC types and features of each subset; the immunologic roles and mechanisms of DC interactions; the roles of DCs in the formation of chronic psoriasis plaques; and the directions for future DC-related research and targeted immunologic therapies in the treatment of psoriasis. 


\section{DC subsets}

In order to gain a better understanding of the immunologic roles of DCs, it is first necessary to illustrate the distinct DC subsets and their defining features as well as specific functions. Widely distributed within the human body, DCs circulate in the bloodstream in immature forms and exist within the vast majority of peripheral tissues under steadystate conditions; following stimulation, DCs migrate to lymphoid organs [6]. DCs are thought to contribute to a local skin-resident immune network: normal skin under resting conditions contains a large pool of memory T-cells (i.e. T-cell numbers within normal skin double the number of circulating T-cells) and APCs, which can respond to antigenic stimuli and effect immunologic responses; it is also believed that keratinocytes secrete cytokines which may play a role in recruiting T-cells to normal as well as inflamed skin; normal skin also contains natural killer (NK) cells, NK T-cells, and macrophages [9, 14]. Several types of DC progenitors have been demonstrated in human peripheral blood: $\mathrm{CD}_{14}{ }^{+}$and $\mathrm{CD} 16^{+}$monocytes; plasmacytoid DCs (pDCs); and Langerhans cell (LC) precursors [15, 27, 54, 60, 65] (Table 1). Blood monocytes are believed to differentiate into either tissue macrophages or myeloid DCs, which are professional APCs $[15,54]$. Within human monocyte subpopulations, $\mathrm{CD} 14^{+}$monocytes predominate and $\mathrm{CD}_{16}{ }^{+}$monocytes are relatively infrequent; these groups differ in the expression profiles of chemokine receptors and adhesion molecules [65]. Another DC subset that can be identified in peripheral blood, pDCs represent an uncommon cell type that morphologically resembles plasma cells [10]. During pro-inflammatory states, pDCs are capable of generating type 1 interferons and migrating to inflamed tissue sites (e.g. cutaneous SLE, contact dermatitis, epidermis and dermis of psoriatic lesions) as well as lymphoid tissues; type 1 interferon family members collectively serve a wide variety of functions including antiviral actions, the prevention of $\mathrm{T}$-cell death, memory $\mathrm{T}$-cell expansion, stimulation of DC maturation, and activation of NK cells [10, 15, 40, 48].

As in the bloodstream, distinct DC subsets have been identified in the peripheral tissues: LCs are found in the epidermis as well as in the stratified squamous epithelium of vaginal and oral mucosa; CD $11 \mathrm{c}^{+} / \mathrm{CD} 11 \mathrm{~b}^{+}$submucosal DCs are located beneath the mucosal epithelium; CD $11 b^{+}$ and $\mathrm{CD}_{11 \mathrm{c}^{+}}$dermal DCs are present under the skin basement membrane [31, 69, 71, 73] (Table 1). LCs, which are recognized as immature DCs, contain characteristic cytoplasmic Birbeck granules and express a variety of cell surface molecules (e.g. Lag, Langerin, CD1a, CD205) [1, 18, 26, 29, 31]. Traditionally, LCs have been recognized as major players in antigen capture, maturation, migration to draining lymph nodes, and subsequent T-cell stimulation through major histocompatibility complex (MHC) proteins. [1, 3, 6, 16, 55, 56, 61]. As proposed by Zhao et al.

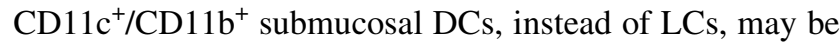
the dominant APCs when vaginal epithelium is infected by certain pathogens such as herpes simplex virus (HSV) type 2 ; in this setting, submucosal DCs migrate to infected regions, phagocytose viral antigen, travel to draining lymph nodes, and present viral peptides to CD4+ T-cells thereby stimulating T-cell activation and cytokine secretion [73]. Aside from anatomic localization, $\mathrm{CD}_{1} 1 \mathrm{~b}^{+}$dermal DCs are functionally similar to their submucosal counterparts [28].

Table 1 Cutaneous dendritic cell types and features of each subset

\begin{tabular}{|c|c|}
\hline Type & Features \\
\hline CD $14^{+}$monocyte & Comprise the majority of monocytes in humans [65]; give rise to tissue macrophages or myeloid DCs [54] \\
\hline $\mathrm{CD}_{16}{ }^{+}$monocyte & Represent an infrequent monocyte population in humans [65]; give rise to DCs [65] \\
\hline Plasmacytoid DC & $\begin{array}{l}\text { Under inflammatory conditions, enter lymph nodes via high endothelial venules and produce type } 1 \text { inter- } \\
\text { ferons [10]; increased numbers in psoriatic lesions [11] }\end{array}$ \\
\hline Langerhans cell & $\begin{array}{l}\text { Contain characteristic intracellular Birbeck granules; immature DC of myeloid lineage [69]; can give rise } \\
\text { to mature DC [69]; present in normal epidermis and psoriatic epidermis [31] }\end{array}$ \\
\hline $\begin{array}{l}\mathrm{CD} 11 \mathrm{c}^{+} / \mathrm{CD} 11 \mathrm{~b}^{+} \\
\text {submucosal } \mathrm{DC}\end{array}$ & $\begin{array}{l}\text { Capable of antigen uptake, T-helper lymphocyte priming, and subsequent cytokine production in pro- } \\
\text { inflammatory states [73] }\end{array}$ \\
\hline $\mathrm{CD}_{11} \mathrm{~b}^{+}$dermal DC & Similar roles as $\mathrm{CD}_{11} \mathrm{~b}^{+}$submucosal DCs [28] \\
\hline CD11c ${ }^{+}$dermal DC & $\begin{array}{l}\text { Immature DC of myeloid lineage [31]; when stimulated, become mature and capable of T-cell activation } \\
\text { [69]; increased numbers in psoriatic skin [11] }\end{array}$ \\
\hline $\begin{array}{l}\text { CD11 } \mathrm{c}^{+} \text {iNOS- and } \\
\text { TNF-producing cells }\end{array}$ & $\begin{array}{l}\text { Similar to Tip-DCs, which are found in mice [35]; increased numbers in epidermis and dermis of psoriatic } \\
\text { lesions [35]; express DC maturation markers CD83 and DC-LAMP [35]; decreased numbers following } \\
\text { efalizumab treatment [35] }\end{array}$ \\
\hline Inflammatory epidermal DC & Epidermal counterpart to $\mathrm{CD} 11 \mathrm{c}^{+}$dermal DC; increased numbers in psoriatic epidermis [11] \\
\hline Interferon-producing killer DC & $\begin{array}{l}\text { Possess properties of NK cells (e.g. NK-activating receptor expression; similar cytokine expression pro- } \\
\text { file); able to process/present antigen and activate T-cells; capable of producing type } 1 \text { IFN, type } 2 \text { IFN, } \\
\text { or IL-12 depending on the activation stimulus [13] }\end{array}$ \\
\hline
\end{tabular}


CD11 ${ }^{+}$dermal DCs, which are recognized as immature DCs, participate in immune surveillance within the dermis; upon maturation, these cells are capable of T-cell activation $[31,69]$. As described by Lowes et al. a specialized type of

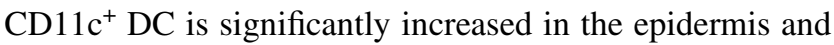
dermis of psoriatic lesions in comparison to normal human skin or uninvolved psoriatic skin; these cells (similar to TNF- and iNOS-producing DC or "Tip DC" in mice) express DC maturation markers (e.g. CD83) as well as the enzyme inducible nitric oxide synthase (iNOS) and increased levels of TNF- $\alpha$ [35]. Classical models of DCs suggest that tissue-resident DCs demonstrate two developmental levels: an immature form functions in antigen capture; a mature form specializes in antigen presentation and T-cell activation in peripheral lymphoid regions as well as in peripheral tissues under pro-inflammatory conditions (e.g. DCs and T-cells may interact within peripheral lymphoid-type areas of psoriatic skin, as described below) (a discussion of DC subsets specific to peripheral lymphoid tissues is beyond the scope of this review). As described in the next section, DC-T-cell interactions depend upon MHC proteins as well as co-stimulatory molecules; targeting this latter mechanism has served as the cornerstone of biologic agents in the treatment of psoriasis.

The recent identification of interferon-producing killer dendritic cells (IKDCs) illustrates the connection between innate and adaptive immune processes; this DC subtype also demonstrates the functional roles of both immature and mature DCs [13]. As described by Chan et al. IKDCs share features common to NK cells (e.g. NK-activating receptor expression, similar cytokines produced) as well as activated DCs. This DC subset is capable of antigen presentation and processing, cytokine (i.e. type 1 and 2 IFN, IL-12) production, T-cell activation, and possible anti-tumor actions [13]. Although initially described in mice, the human IKDC counterpart has yet to be identified; characterization of this versatile DC subtype in humans may hold key implications in directing therapies against cancers and autoimmune diseases such as psoriasis. Aside from anatomic location and cell surface markers, DCs can be further classified based on maturation status.

\section{Immunologic roles of DCs}

While present in the blood and peripheral tissues in an immature form, DCs are thought to be mature following stimulation and migration to secondary lymphoid tissues [15]. The molecular changes demonstrated in the maturation process correlate with the DC transition from cells predominantly involved in antigen detection and uptake to professional APCs (Table 2). In contributing to this latter role, DCs undergo a wide variety of modifications in becoming mature: increased expression of costimulatory molecules (CD40, CD80, CD86) and adhesion molecules (VLA-4 and ICAM-1); the expression of CD83 and DCLysosomal-Associated Membrane Protein (DC-LAMP), which are markers of DC maturation; upregulation of surface MHC molecules; decreased expression of proinflammatory chemokine receptors and a corresponding upregulation of lymphoid chemokine receptors (CCR7); and downregulation of phagocytic and endocytic receptors $[6,11,15,18,51,75]$. DC maturation can be influenced by a number of factors including DC interactions with components of the innate immune system (TLR ligands, NK cells), pro-inflammatory cytokine secretion, heat shock proteins (HSPs), chemotaxins, chemokines, and costimulatory molecule interactions; within the context of psoriatic lesions, the specific antigen initiating the cascade of DC maturation and downstream T-cell activation has yet to be identified (Table 3).

The DC maturation process contributes to the eventual activation of naïve T-cells along with the generation of antigen-specific memory T-cells; this pathway begins with

Table 2 Immature versus mature dendritic cells: differences in surface markers and functional roles

\begin{tabular}{|c|c|c|}
\hline & Markers & Functions \\
\hline Immature & $\begin{array}{l}\text { Express CD1a [18, 26], lectin receptors [18], Toll-like receptors [28], } \\
\text { natural killer (NK)-activating receptors (in the case of } \\
\text { interferon-producing killer DCs) [13], pro-inflammatory } \\
\text { chemokine receptors [15], intracellular MHC class II molecules [68] }\end{array}$ & $\begin{array}{l}\text { Antigen capture via endocytosis, } \\
\text { macropinocytosis, phagocytosis [18]; } \\
\text { antigen processing [68] }\end{array}$ \\
\hline Mature & $\begin{array}{l}\text { Co-stimulatory proteins (CD40, CD80, CD86) [44, 51], CD83 [33], } \\
\text { DC-LAMP, increased surface MHC levels [18], upregulated cell } \\
\text { adhesion molecules (e.g. intercellular adhesion molecule-1) [11] }\end{array}$ & $\begin{array}{l}\text { Lack of phagocytic function [18]; } \\
\text { presence in secondary lymphoid } \\
\text { tissues (DC migration is facilitated by } \\
\text { the upregulation of lymphoid } \\
\text { chemokine receptors) [15]; } \\
\text { antigen presentation via MHC-peptide } \\
\text { complexes [68]; binding and } \\
\text { stimulating T-cells [43]; cytokine } \\
\text { production and secretion [22] }\end{array}$ \\
\hline
\end{tabular}


Table 3 Regulation of dendritic cell activities

\begin{tabular}{ll}
\hline & Activating stimuli \\
\hline $\begin{array}{l}\text { Antigen uptake } \\
\text { and processing }\end{array}$ & (1) Microbial products (e.g. CpG motifs, lipopolysaccharide) stimulate pattern-recognition receptors (i.e. \\
lectin receptors, Toll-like receptors) and subsequent antigen internalization [18, 28, 67] \\
(2) Antigen processing occurs through proteolysis by endocytic proteases, and results in the generation of \\
peptides that are loaded on to MHC molecules [18] \\
(1) Pro-inflammatory chemokines, leukotrienes, cytokines, and other chemotaxins (e.g. C5a and PAF) \\
facilitate DC migration to inflammatory sites [18, 45, 63] \\
(2) Up-regulation of lymphoid chemokine receptors (CCR7) and down-regulation of pro-inflammatory \\
chemokine receptors correlate with DC migration to secondary lymphoid tissues [15] \\
(1) DCs produce IL-12p70, which helps generate T-helper cell type 1 (Th1) responses [51]; IL-12p70 acts \\
as a "third signal", while enhancing CD8+ T-cell survival, clonal expansion, and effector functions [17]; \\
other possible stimulatory cytokines include IL-15, IL-18, IL-23, and IFN- $\alpha$ [22, 36] \\
(2) Mature DCs exhibit surface MHC-peptide complexes for presentation to T-cells [18] \\
(3) Co-stimulatory signals (CD80 and CD86) and adhesion molecules (DC intercellular adhesion molecule- \\
1 binds to T-cell leukocyte-function-associated antigen-1) facilitate DC-T-cell interactions [52] \\
(4) Heat shock proteins and their corresponding receptors (CD91) stimulate: the upregulation of DC co- \\
stimulatory molecules; DC activation of T-cells; DC production of cytokines, which induce T-helper cell \\
proliferation [8, 11] \\
(5) Cross-presentation and priming of cytotoxic T-lymphocytes [43]
\end{tabular}

antigenic stimulation, which occurs through DC detection and uptake of particles. DCs express distinct molecules that may assist in trafficking to sites of inflammation: proinflammatory chemokine receptors (CCR1, CCR5, and CCR6); and receptors for platelet-activating factor (PAF) and $\mathrm{C} 5 \mathrm{a}$, which represent chemotaxins often found at inflammatory sites (e.g. psoriatic lesions) [18, 45, 63]. While proposed to be an important link between the innate and adaptive immune systems in the generation of antimicrobial responses, Toll-like receptors (TLRs) are expressed by numerous cell types including DCs, neutrophils, NK cells, mast cells, eosinophils, monocytes, mucosal epithelial cells, macrophages, and keratinocytes [25, 28, 32]. Collectively, TLRs are able to detect a variety of viral and bacterial components (e.g. unmethylated CpG DNA, proteoglycans, and lipopolysaccharide) [28, 67]. Further complicating the picture is that distinct DC subsets vary in the TLRs that are expressed [28]. In light of their roles in DC maturation, TLRs are recognized as key participants in the generation of downstream T-cell responses. Within the context of autoimmune disease, TLR-associated pathways activate the transcription factor nuclear factor kappa B $(\mathrm{NF}-\kappa \mathrm{B})$ with the eventual transcription of genes encoding pro-inflammatory cytokines and proteins with microbicidal effects [39].

Following antigen recognition at peripheral sites, immature DCs perform antigen capture and processing through mechanisms including receptor-mediated endocytosis, phagocytosis, and macropinocytosis [18]. Once antigens are captured and within the cell, processing occurs within endosomes as the molecules are degraded into peptides that are subsequently complexed with MHC class II molecules.
Immature DCs contain large numbers of intracellular MHC class II rich compartments; as maturation takes place, MHC-peptide complexes are exported to the cell membrane for eventual antigen presentation to T-cells [68]. MHC class II presentation, while limited to macrophages, DCs, and activated B- and T-cells, is also recognized as exogenous (i.e. presented peptides originate from extracellular proteins); this pathway helps present extracellular pathogens [68]. On the other hand, MHC class I presentation involves all nucleated cells, protects against intracellular pathogens, and functions through an endogenous pathway: intracellular proteins are degraded in the proteasome and released into the cytosol; these cytosolic proteins are transported to the endoplasmic reticulum, where they are complexed with MHC class I molecules for subsequent exportation to the cell surface [68]. MHC class I and II molecules are utilized for presentation to CD8+ and CD4+ T-cells, respectively. MHC class I harbors the major psoriasis susceptibility locus PSORS1, which is present in approximately $10 \%$ of psoriasis cases [23]. MHC class I has also been associated with psoriasis susceptibility alleles such as HLA-Cw $\times 0602$, through which homozygotes have a relative risk of 23.1 for developing disease and heterozygotes have a relative risk of 8.9; recent evidence indicates that there is considerable overlap between HLA-Cw alleles and the PSORS1 locus especially among patients with early-onset psoriasis [2, 22, 46, 51].

Following antigen uptake and processing, DCs migrate to secondary lymphoid tissues for presentation to T-cells. DC trafficking to draining lymph nodes is orchestrated through a complex interplay of pro-inflammatory cytokines (TNF- $\alpha$, IL-1 beta, IL-18), chemokine and chemokine 
receptors (CCL19, CCR2, CCR6, CCR7), leukotrienes, and cell adhesion molecules (e.g. E-cadherin down-regulation); this process corresponds with a TLR-dependent downregulation of pro-inflammatory chemokine receptors and up-regulation of lymphoid chemokine receptors [11, 15, 69, 72] (Table 3). Psoriatic lesions may serve as potential sites for chronic T-cell activation through lymphoid-organizing chemokines that are found at increased levels in psoriatic lesions [33]. It has been proposed that these chemokines are capable of organizing DCs and T-cells into lymphoid-type tissues that can possibly support ongoing, peripheral APCT-cell interactions that are usually confined to the lymph nodes or spleen [33]. For example, psoriatic skin demonstrates elevated expression of a ligand (CCL20/MIP-3 alpha) for the pro-inflammatory chemokine receptor CCR6; as CCR6 is present on immature DCs as well as on memory T-cells, it is thought that these cell types migrate to psoriatic lesions while subsequently interacting and thereby generating downstream inflammatory-type responses [15, 24].

In arriving at the T-cell areas of lymphoid tissues, mature DCs interact with T-cells in a number of ways. These mechanisms include: antigen-specific interaction through $\mathrm{T}$-cell receptor association with the $\mathrm{MHC}$-peptide complex on the DC surface; costimulatory signals involving CD80 and CD86 which are expressed by mature DCs and that are recognized by CD28 expressed on T-cells; other costimulatory interactions (e.g. CD2:LFA-3, LFA1:ICAM-1) between T-cells and APCs; DC presentation of non-peptide molecules (e.g. lipids) to T-cells through CD1mediated pathways; CD40-CD154 interactions; and crosspresentation [26, 28, 43, 44, 49, 51]. Within the context of psoriatic plaques, HSPs and their corresponding receptors (i.e. CD91) influence DC activation of T-cells; certain HSPs possess the ability to upregulate DC costimulatory molecules, and also stimulate DC production of IL-12, which triggers $\mathrm{T}$-helper cell proliferation [8, 11]. DC licensing by helper T-cells involves the expression of CD40 and CD154 by DCs and T-cells, respectively. As suggested by Miga et al. CD40-CD154 associations are essential for sustaining mature DC and T-cell populations, as well as promoting T-cell proliferation following APC-T-cell interactions [44]. In highlighting the importance of this system in cell-mediated immunity, Smith et al. propose that DC licensing is integral for generating and maintaining primary and memory cytotoxic T-lymphocyte (CTL) responses [62]. With regards to cell-mediated immunity, DCs also possess the unique ability to cross-present antigens to CD8+ CTLs. Through this mechanism, DCs present proteins derived from the uptake and processing of extracellular antigens (e.g. tumor or virus-infected cells) and subsequently stimulate CTLs; cross-presentation involves pathogens that are unable to invade DCs and gain access to endogenous MHC class I mechanisms [43]. In contrast to cross-presentation, the involvement of self-antigens may alternately lead to the attenuation of CTL responses (i.e. tolerance) through distinct mechanisms: circulating T-lymphocytes may fail to recognize self-antigens; the removal of self-reactive T-cells through apoptotic mechanisms; impaired downstream responses following $\mathrm{T}$-cell receptor binding, such as in the absence of co-stimulatory interactions [43]. Several factors (e.g. DC activation state, antigen levels, duration of DC-T-cell interaction) determine whether T-cell priming or tolerance occurs; for example, immature DCs lack appropriate costimulatory signals and lead to peripheral tolerance [43]. It has been suggested that cross-presentation of certain foreign antigens (e.g. streptococcal proteins), subsequent CD8+ T-cell priming, and disturbances of regulatory mechanisms lead to potentially self-perpetuating lesions present in chronic plaque psoriasis [22].

\section{DCs and psoriasis plaques}

The interactions between DCs and T-cells, and associated downstream responses play essential roles in the formation of chronic psoriasis plaques. Recent studies have demonstrated a high number of pDCs, immature as well as mature DCs, CD11c ${ }^{+}$iNOS-and TNF-producing cells, and inflammatory dendritic epidermal cells (i.e. the epidermal counterpart to CD11c ${ }^{+}$dermal DCs) within diseased skin [11, 35]. pDCs are also increased in uninvolved skin of individuals with psoriasis, and may play a role in the subsequent development of lesions [48]. With regards to functional capabilities, dermal DCs within psoriatic lesions are stronger stimulators of T-cell proliferation than are DCs derived from peripheral blood or dermal DCs from the skin of healthy patients [47]. It has been proposed that the preferential association between DCs and psoriatic epidermal CD4+ T-cells may lead to the stimulation and subsequent clonal expansion of epidermal CD8+ T-cells; along with CD4+ T-cells and DCs, CD8+ T-cells are key players in the production of pro-inflammatory cytokines that have been implicated in psoriasis $[22,50,53]$. Along these lines, the AGR129 mouse xenograft model has suggested that the localized expansion of T-cells along with downstream cytokine production play important roles in the development of psoriatic plaques [7, 51]. Local skin-resident immune cells (e.g. DCs, T-cells) and associated cytokines are present in the uninvolved skin of psoriasis patients, and may be sufficient to initiate/maintain lesions following inciting triggers [9].

An extensive cytokine network generated by activated DCs and T-cells mediates the formation of psoriasis lesions. Collectively, DCs have been shown to secrete numerous cytokines that are implicated in psoriasis including 
TNF- $\alpha$, IFN- $\alpha$, IL-12, IL-23, and IL-15 [22, 36]. IFN- $\alpha$, which is produced by pDCs that are present within psoriasis plaques, plays a key role in the stimulation and proliferation of T-cells as well as the formation of psoriatic skin [48]. IL-12 and IL-23 trigger T-helper cell activation and associated downstream responses within the context of the type 1 pathway in psoriasis [51]. As suggested by Zheng et al. IL-23 activates T-helper cells that subsequently produce IL-17 and IL-22; this latter cytokine contributes to dermal inflammation and epidermal hyperplasia characteristic of psoriasis [74]. Similarly, IL-15 is a pro-inflammatory cytokine that induces $\mathrm{T}$-cell proliferation as well as skin hyperplasia $[11,57,70]$. As noted previously in this paper, activated T-cells secrete IFN- $\gamma$ and TNF- $\alpha$, which are essential players in the potentially self-perpetuating type 1 pathway of psoriasis. In light of the immense cytokine network described, there exist numerous possible targets for anti-psoriatic immunologic therapies.

\section{DCs, T-cells, and anti-psoriatic immunotherapies}

In targeting the mechanisms involving DCs, T-cells, and cytokines within psoriatic lesions, immunologic therapies have been successful in reducing disease severity. The immunologic synapse (i.e. the platform for APC-T-cell interactions) has been an effective focus for therapy as suggested by several key examples: psoralen and ultraviolet A (PUVA) treatment resulted in decreased numbers of lesional DCs as well as epidermal T-cells in association with the clearing of chronic plaque psoriasis; cyclosporine treatment applied to plaque psoriasis resulted in reduced numbers of epidermal and dermal T-cells as well as improvements in disease-related symptoms; alefacept (a CD2-binding LFA-3 Ig fusion protein) has been proposed to clear psoriasis lesions through the depletion of CD2expressing cells (e.g. lesional T-cells and DCs); efalizumab (a human monoclonal antibody directed against LFA-1), which blocks the interaction between leukocyte-functionassociated antigen-1 (LFA-1) on T-cells and intercellular adhesion molecule-1 (ICAM-1) on DCs, is also effective in clearing psoriatic lesions as well as in reducing $\mathrm{CD} 11 \mathrm{c}^{+} \mathrm{DC}$ numbers $[4,5,12,36,52]$. These findings suggest that the mechanisms behind psoriasis depend upon interactions between APCs and T-cells; blocking components of this synapse could potentially minimize downstream responses and disease severity. In addition to therapies targeting the interactions between APCs and T-cells, emerging anti-psoriatic medications aim to modify cytokine responses in the context of the type 1 pathway. TNF- $\alpha$ antagonists such as infliximab and etanercept result in a rapid clearing of psoriatic lesions [20, 41, 59]. Aside from their pro-apoptotic roles, TNF- $\alpha$ antagonists may act upon distinct inflamma- tory pathways in psoriatic lesions; etanercept is associated with decreases in pro-inflammatory cytokines and reduced infiltration of cells characteristically involved in plaques [21, 37, 38]. Another cytokine-focused treatment strategy has involved the administration of IL-10 and other Th2 molecules (e.g. IL-4, IL-11) in an attempt to increase the ratio of Th2:Th1 cytokines; this would presumably attenuate the role of the type 1 pathway in psoriatic lesions [51]. IL-12, IL-15 and IL-23 also serve as promising targets for anti-psoriatic therapies, as each of these cytokines has been implicated in the type 1 pathway and in stimulating downstream cytokine production [31]. A phase 1 study evaluated a humanized monoclonal antibody against the p40 subunit of human IL-12; anti-IL-12p40 was well-tolerated and effectively cleared psoriatic lesions [30]. As additional cytokines and costimulatory molecules are linked to the pathogenesis of psoriatic lesions, immunologic therapies will have an increased number of targets upon which to act.

\section{Summary}

In targeting DC-T-cell interactions and associated cytokine networks, immunologic therapies have generally been effective in the treatment of psoriasis. As DCs are central players in the potentially self-sustaining type 1 inflammatory network, it is of great interest to further define DC characteristics (e.g. DC life cycle, functional roles). DCs also possess unique immunologic characteristics (e.g. cross-presentation, linking innate and adaptive processes) that can be exploited in the development of additional biologic response therapies. The identification of novel DC subsets as well as further characterizing existing "players" (e.g. IKDCs) will help achieve a greater understanding of psoriasis and other chronic inflammatory conditions. DCs serve a key role in the immunopathogenetic mechanisms surrounding psoriasis and in the formation as well as sustenance of chronic plaques.

\section{References}

1. Allan RS et al (2003) Epidermal viral immunity induced by $\mathrm{CD} 8 \alpha^{+}$dendritic cells but not by langerhans cells. Science 301:1925-1928

2. Allen MH et al (2005) The major susceptibility locus PSORS1 is not a risk factor for late-onset psoriasis. J Invest Dermatol 124(1):103-106

3. Austyn JM (1996) New insights into the mobilization and phagocytic activity of dendritic cells. J Exp Med 183(4):1287-1292

4. Baker BS et al (1985) Epidermal T lymphocytes and dendritic cells in chronic plaque psoriasis: the effects of PUVA treatment. Clin Exp Immunol 61(3):526-534

5. Baker BS et al (1987) The effects of cyclosporin A on T lymphocyte and dendritic cell sub-populations in psoriasis. Br J Dermatol 116(4):503-510 
6. Banchereau J, Steinman RM (1998) Dendritic cells and the control of immunity. Nature 392(6673):245-252

7. Boyman $\mathrm{O}$ et al (2004) Spontaneous development of psoriasis in a new animal model shows an essential role for resident T-cells and tumor necrosis factor-\{alpha\}. J Exp Med 199:731-736

8. Boyman $\mathrm{O}$ et al (2005) Activation of dendritic antigen-presenting cells expressing common heat shock protein receptor CD91 during induction of psoriasis. Br J Dermatol 152(6):1211-1218

9. Boyman $\mathrm{O}$ et al (2007) The pathogenic role of tissue-resident immune cells in psoriasis. Trends Immunol 28(2):51-57

10. Cella $\mathrm{M}$ et al (1999) Plasmacytoid monocytes migrate to inflamed lymph nodes and produce large amounts of type I interferon. Nat Med 5:919-923

11. Chamian F, Krueger JG (2004) Psoriasis vulgaris: an interplay of T-lymphocytes, dendritic cells, and inflammatory cytokines in pathogenesis. Curr Opin Rheumatol 16:331-337

12. Chamian $\mathrm{F}$ et al (2005) Alefacept reduces infiltrating T-cells, activated dendritic cells, and inflammatory genes in psoriasis vulgaris. Proc Natl Acad Sci USA 102(6):2075-2080

13. Chan CW et al (2006) Interferon-producing killer dendritic cells provide a link between innate and adaptive immunity. Nat Med 12(2):207-213

14. Clark RA et al (2006) The vast majority of CLA ${ }^{+} \mathrm{T}$-cells are resident in normal skin. J Immunol 176(7):4431-4439

15. Cravens PD, Lipsky PE (2002) Dendritic cells, chemokine receptors, and autoimmune inflammatory diseases. Immunol Cell Biol 80:497-505

16. Cumberbatch M, Kimber I (1995) Tumour necrosis factor-alpha is required for accumulation of dendritic cells in draining lymph nodes and for optimal contact sensitization. Immunology 84(1):31-35

17. Curtsinger JM et al (2003) CD8 T-Cell clonal expansion and development of effector function require prolonged exposure to antigen, costimulation, and signal 3 cytokine. J Immunol 171(10):5165-5171

18. Cutler CM et al (2001) Dendritic cells: immune Saviors or Achilles' heel? Infect Immun 69(8):4703-4708

19. Fierlbeck G et al (1990) Psoriasis induced at the injection site of recombinant interferon gamma. Results of immunohistologic investigations. Arch Dermatol 1990 126:351-355

20. Gottlieb AB et al (2003) Infliximab monotherapy provides rapid and sustained benefit for plaque-type psoriasis. J Am Acad Dermatol 48(6):829-835

21. Gottlieb AB et al (2005) TNF inhibition rapidly down-regulates multiple pro-inflammatory pathways in psoriasis plaques. J Immunol 175(4):2721-2729

22. Gudjonsson JE et al (2004) Immunopathogenic mechanisms in psoriasis. Clin Exp Immunol 135:1-8

23. Helms Cet al (2005) Localization of PSORS1 to a haplotype block harboring HLA-C and distinct from corneodesmin and HCR. Hum Genet 118:466-476

24. Homey BM et al (2000) Upregulation of macrophage inflammatory protein-3 alpha/CCL20 and CC chemokine receptor 6 in psoriasis. J Immunol 164:6621-6632

25. Hornung V et al (2002) Quantitative expression of toll-like receptor 1-10 mRNA in cellular subsets of human peripheral blood mononuclear cells and sensitivity to $\mathrm{CpG}$ oligodeoxynucleotides. J Immunol 168:4531-4537

26. Hunger RE et al (2004) Langerhans cells utilize CD1a and langerin to efficiently present nonpeptide antigens to T-cells. J Clin Invest 113:701-708

27. Ito T et al (1999) A CD1a+/CD11c+ subset of human blood dendritic cells is a direct precursor of Langerhans cells. J Immunol 163:1409-1419

28. Iwasaki A, Medzhitov R (2004) Toll-like receptor control of the adaptive immune responses. Nat Immunol 5(10):987-995
29. Kashihara $\mathrm{M}$ et al (1986) A monoclonal antibody specifically reactive to human Langerhans cells. J Invest Dermatol 87:602-607

30. Kauffman CL et al (2004) A phase I study evaluating the safety, pharmacokinetics, and clinical response of a human IL-12 p40 antibody in subjects with plaque psoriasis. J Invest Dermatol 123(6): 1037-1044

31. Krueger JG, Bowcock A (2005) Psoriasis pathophysiology: current concepts of pathogenesis. Ann Rheum Dis 64:ii30-ii36

32. Lebre MC et al (2007) Human keratinocytes express functional Toll-like receptor 3,4,5, and 9. J Invest Dermatol 127(2):331-341

33. Lew W et al (2004) Psoriasis vulgaris: cutaneous lymphoid tissue supports T-cell activation and "Type 1" inflammatory gene expression. Trends Immunol 25(6):295-305

34. Lowes MA et al (2004) Current concepts in the immunopathogenesis of psoriasis. Clin Dermatol 22(4):349-369

35. Lowes MA et al (2005) Increase in TNF- $\alpha$ and inducible nitric oxide synthase-expressing dendritic cells in psoriasis and reduction with efalizumab (anti-CD11a). PNAS 102(52):19057-19062

36. Lowes MA et al (2007) Pathogenesis and therapy of psoriasis. Nature 445(7130):866-873

37. Malaviya $\mathrm{R}$ et al (2006) Etanercept induces apoptosis of dermal dendritic cells in psoriatic plaques of responding patients. J Am Acad Dermatol 55(4):590-597

38. Malaviya $R$ et al (2006) Induction of lesional and circulating leukocyte apoptosis by infliximab in a patient with moderate to severe psoriasis. J Drugs Dermatol 5(9):890-893

39. McInturff JE et al (2005) The role of toll-like receptors in the pathogenesis and treatment of dermatological disease. J Invest Dermatol 125(1):1-8

40. McKenna K et al (2005) Plasmacytoid dendritic cells: linking innate and adaptive immunity. J Virol 79(1):17-27

41. Mease PJ et al (2000) Etanercept in the treatment of psoriatic arthritis and psoriasis: a randomized trial. Lancet 356(9227):385390

42. Mehlis SL, Gordon KB (2003) The immunology of psoriasis and biologic immunotherapy. J Am Acad Dermatol 49:S44-S50

43. Melief CJ (2003) Regulation of cytotoxic T lymphocyte responses by dendritic cells: peaceful coexistence of cross-priming and direct priming? Eur J Immunol 33:2645-2654

44. Miga AJ et al (2001) Dendritic cell longevity and T-cell persistence is controlled by CD154-CD40 interactions. Eur J Immunol 31:959-965

45. Mrowietz U et al (2001) Psoriasis scales contain C5a as the predominant chemotaxin for monocyte-derived dendritic cells. Exp Dermatol 10(4):238-245

46. Nair RP et al (2006) Sequence and haplotype analysis supports HLA-C as the psoriasis susceptibility 1 gene. Am J Hum Genet 78(5):827-851

47. Nestle FO et al (1994) Characterization of dermal dendritic cells in psoriasis: autostimulation of T lymphocytes and induction of Th1 type cytokines. J Clin Invest 94:202-209

48. Nestle FO et al (2005) Plasmacytoid predendritic cells initiate psoriasis through interferon-alpha production. J Exp Med 202(1):135-143

49. Nickoloff BJ et al (1993) Activated keratinocytes present bacterial-derived superantigens to $\mathrm{T}$ lymphocytes: relevance to psoriasis. J Dermatol Sci 6:127-133

50. Nickoloff BJ, Wrone-Smith T (1999) Injection of pre-psoriatic skin with CD4+ T-cells induces psoriasis. Am J Pathol 155:145158

51. Nickoloff BJ, Nestle FO (2004) Recent insights into the immunopathogenesis of psoriasis provides new therapeutic opportunities. J Clin Invest 113:1664-1675

52. Nickoloff BJ, Stevens SR (2006) What have we learned in dermatology from the biologic therapies? J Am Acad Dermatol S4(3):S143-S149 
53. Paukkonen K et al (1992) The development of manifest psoriatic lesions is linked with the invasion of CD8+ T-cells and CD11c+ macrophages into the epidermis. Arch Dermatol Res 284:375-379

54. Randolph GJ et al (1998) Differentiation of monocytes into dendritic cells in a model of transendothelial trafficking. Science 282:480-483

55. Roake JA et al (1995) Dendritic cell loss from nonlymphoid tissues after systemic administration of lipopolysaccharide, tumor necrosis factor, and interleukin 1. J Exp Med 181:2237-2247

56. Romani $\mathrm{N}$ et al (1989) Presentation of exogenous protein antigens by dendritic cells to T-cell clones. Intact protein is presented best by immature, epidermal Langerhans cells. J Exp Med 169:1169-1178

57. Ruckert R et al (2000) Inhibition of keratinocyte apoptosis by IL15: a new parameter in the pathogenesis of psoriasis? J Immunol 165(4):2240-2250

58. Schon MP, Ruzicka T (2001) Psoriasis: the plot thickens. Nat Immunol 2:91

59. Schopf RE et al (2002) Treatment of psoriasis with the chimeric monoclonal antibody against tumor necrosis factor alpha, infliximab. J Am Acad Dermatol 46:886-891

60. Siegal FP et al (1999) The nature of the principal type 1 interferonproducing cells in human blood. Science 284:1835-1837

61. Silberberg-Sinakin I et al (1976) Antigen-bearing langerhans cells in skin, dermal lymphatics and in lymph nodes. Cell Immunol 25(2):137-151

62. Smith CM et al (2004) Cognate CD4+ T-cell licensing of dendritic cells in CD8+ T-cell immunity. Nat Immunol 5(11):1143-1148

63. Sozzani S et al (2000) Chemokines and dendritic cell traffic. J Clin Immunol 20:151-160
64. Steinman RM, Cohn ZA (1973) Identification of a novel cell type in peripheral lymphoid organs of mice. J Exp Med 137:1142-1162

65. Tacke F, Randolph GJ (2006) Migratory fate and differentiation of blood monocyte subsets. Immunobiology 211(6-8):609-618

66. Taieb J et al (2006) A novel dendritic cell subset involved in tumor immunosurveillance. Nat Med 12(2):214-219

67. Takeda K et al (2003) Toll-like receptors. Ann Rev Immunol 21:335-376

68. Trombetta ES, Mellman I (2005) Cell biology of antigen processing in vitro and in vivo. Ann Rev Immunol 23:975-1028

69. Valladeau J, Saeland S (2005) Cutaneous dendritic cells. Semin Immunol 17:273-283

70. Villadsen LS et al (2003) Resolution of psoriasis upon blockade of IL-15 biological activity in a xenograft mouse model. J Clin Invest 112(10):1571-1580

71. Wan H, Dupasquier M (2005) Dendritic cells in vivo and in vitro. Cell Mol Immunol 2(1):28-35

72. Winzler C et al (1997) Maturation stages of mouse dendritic cells in growth factor-dependent long-term cultures. J Exp Med $185: 317-328$

73. Zhao X et al (2003) Vaginal submucosal dendritic cells, but not Langerhans cells, induce protective Th1 responses to herpes simplex virus-2. J Exp Med 197(2):153-162

74. Zheng $\mathrm{Y}$ et al (2007) Interleukin-22, a TH17 cytokine, mediates IL-23-induced dermal inflammation and acanthosis. Nature 445:648-651

75. Zhou LJ, Tedder TF (1996) CD14+ blood monocytes can differentiate into functionally mature CD83+ dendritic cells. Proc Natl Acad Sci USA 93:2588-2592 